\title{
A NOTE ON REAL INTERPOLATION OF HARDY SPACES IN THE POLYDISK
}

\author{
BJÖRN JAWERTH AND ALBERTO TORCHINSKY ${ }^{1}$
}

\begin{abstract}
The atomic decomposition for the Hardy spaces $H^{p}$ of the product of upper half-spaces is used to characterize Peetre's $K$-functional for two such spaces.
\end{abstract}

0. Introduction. We present an application of the atomic decomposition for the Hardy spaces $H^{p}$ of the product of upper half-spaces. Our main results are the computation of Peetre's $K$-functional for two such spaces and the fact that these spaces are amenable to the method of real interpolation. We also include some applications to Hardy's inequality (for the Fourier transform) and to fractional integration.

1. The $K$-functional. In order to simplify the presentation of the results, we work exclusively with the domain $\mathbf{R}_{+}^{2} \times \mathbf{R}_{+}^{2}$ and its distinguished boundary $\mathbf{R}^{2}$. We will use the same definitions and notations as in R. Fefferman and S. Y. A. Chang $[\mathbf{1}, \mathbf{2}]$. Points in $\mathbf{R}_{+}^{2} \times \mathbf{R}_{+}^{2}$ are denoted by $(y, t)$, where $y=\left(y_{1}, y_{2}\right) \in \mathbf{R}^{2}$ and $t=\left(t_{1}, t_{2}\right), t_{1}, t_{2}>0$. We reserve the notation $\varphi(u)$ for an even, real-valued, $C^{\infty}(\mathbf{R})$ function supported in $[-1,1]$ such that

$$
\int_{0}^{\infty} \hat{\varphi}(u)^{2} \frac{d u}{u}=1, \quad \text { and } \quad\left(\frac{d}{d u}\right)^{m} \hat{\varphi}(0)=0,
$$

for sufficiently large $m$ to be specified. With this function $\varphi$ we associate the two-parameter dilation

$$
\Phi_{t}(y)=\frac{\varphi\left(y_{1} / t_{1}\right) \varphi\left(y_{2} / t_{2}\right)}{t_{1} t_{2}}, \quad t_{1}, t_{2}>0
$$

defined on $\mathbf{R}^{2}$. For a tempered distribution $f \in S^{\prime}\left(\mathbf{R}^{2}\right)$ we put $f(y, t)=f * \Phi_{t}(y)$. Further, if $x=\left(x_{1}, x_{2}\right) \in \mathbf{R}^{2}, \Gamma(x)$ denotes the product cone $\Gamma\left(x_{1}\right) \times \Gamma\left(x_{2}\right)$, or

$$
\Gamma(x)=\left\{(y, t):\left|x_{1}-y_{1}\right|<t_{1},\left|x_{2}-y_{2}\right|<t_{2}\right\} .
$$

We can now introduce the double $S$-function of $f$ defined by

$$
S f(x)=\left(\iint_{\Gamma(x)}|f(y, t)|^{2} d y \frac{d t_{1} d t_{2}}{t_{1}^{2} t_{2}^{2}}\right)^{1 / 2} .
$$

It is a known fact that for $m \geq 0$ and $1<p<+\infty$,

$$
\|S f\|_{L^{p}\left(\mathbf{R}^{2}\right)} \leq C_{p}\|f\|_{L^{p}\left(\mathbf{R}^{2}\right)} .
$$

Received by the editors February 19, 1985.

1980 Mathematics Subject Classification. Primary 42B30; Secondary 46E30.

${ }^{1}$ The authors were supported in part by grants from the National Science Foundation. 


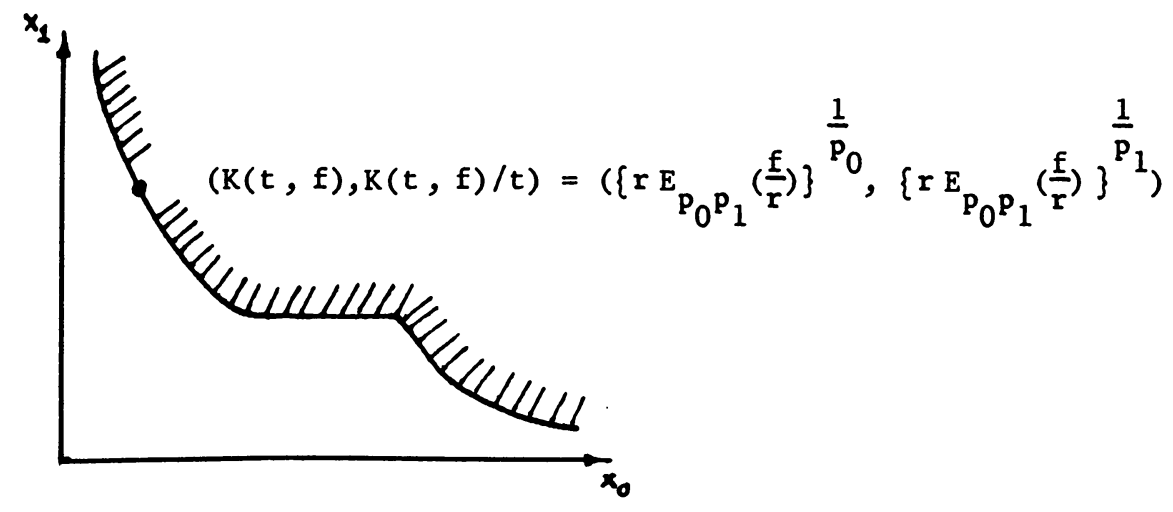

FIGURE 1

It is also well known that we can introduce the Hardy spaces $H_{q}^{p}\left(\mathbf{R}^{2} \Varangle \mathbf{R}^{2}\right)$ as $\left\{f \in S^{\prime}\left(\mathbf{R}^{2}\right):\|S f\|_{L^{p}\left(\mathbf{R}^{2}\right)}<+\infty\right\}$ (modulo a simple normalization). Here $0<$ $p<+\infty$ and $\|S f\|_{L^{p}}$, with $m \sim(1 / p-1)$ for $0<p \leq 1$, gives one of the possible equivalent $H^{p}$ "norms" $\|f\|_{H^{p}}$. In fact, the class $H^{p}$ is independent of the choice of $\Phi$ (see Gundy-Stein [5] and Merryfield [9]).

Finally, we recall the definition of the $K$-functional. Given $f \in H^{p_{0}}+H^{p_{1}}$ and $t>0$, put

$$
K\left(t, f ; H^{p_{0}}, H^{p_{1}}\right)=\inf _{f=f_{0}+f_{1}} \max \left(\left\|f_{0}\right\|_{H^{p_{0}}}, t\left\|f_{1}\right\|_{H^{p_{1}}}\right) .
$$

A similar definition holds for two $L^{p}$ spaces. We have

THEOREM A. Let $0<p_{0}<p_{1}<+\infty$. Then

$$
K\left(t, f ; H^{p_{0}}, H^{p_{1}}\right) \approx K\left(t, S f ; L^{p_{0}}, L^{p_{1}}\right) .
$$

PROOF. From the subadditivity of the $S$-function it follows at once that

$$
K\left(t, S f ; L^{p_{0}}, L^{p_{1}}\right) \leq C K\left(t, f ; H^{p_{0}}, H^{p_{1}}\right) .
$$

To prove the converse, we put

$$
E_{p_{0} p_{1}}\left(f ; H^{p_{0}}, H^{p_{1}}\right)=\inf _{f=f_{0}+f_{1}} \max \left(\left\|f_{0}\right\|_{H^{p_{0}}}^{p_{0}},\left\|f_{1}\right\|_{H^{p_{1}}}^{p_{1}}\right),
$$

and similarly for $L^{p_{0}}, L^{p_{1}}$. By looking at the Gagliardo diagram $\Gamma(f)=\left\{\left(x_{0}, x_{1}\right) \in\right.$ $\mathbf{R}^{2}: f=f_{0}+f_{1}$ with $\left.\left\|f_{0}\right\|_{H^{p_{0}}} \leq x_{0},\left\|f_{1}\right\|_{H^{p_{1}}} \leq x_{1}\right\}$ (see Figure 1), we easily see that the right continuous inverse of $\left\{E_{p_{0} p_{1}}\left(f / t ; H^{p_{0}}, H^{p_{1}}\right)\right\}^{p_{0} p_{1} /\left(p_{1}-p_{0}\right)}$ (as a function of $t$ ) is $K\left(t, f ; H^{p_{0}}, H^{p_{1}}\right) / t^{p_{1} /\left(p_{1} p_{0}\right)}$ and similarly for $\bar{L}^{p_{0}}, L^{p_{1}}$ (cf. Jawerth et al. [6]). Now,

$$
\begin{aligned}
E_{p_{0} p_{1}}\left(\frac{f}{c} ; L^{p_{0}}, L^{p_{1}}\right) & \leq \int_{|f|>1}|f|^{p_{0}} d x+\int_{|f| \leq 1}|f|^{p_{1}} d x \\
& \leq E_{p_{0} p_{1}}\left(c f ; L^{p_{0}}, L^{p_{1}}\right)
\end{aligned}
$$

for some constant $c$ independent of $f$. Hence, to prove the inequality opposite to (1.1), we need to show that

$$
E_{p_{0} p_{1}}\left(f ; H^{p_{0}}, H^{p_{1}}\right) \leq C\left\{\int_{S f>1} S f(x)^{p_{0}} d x+\int_{S f \leq 1} S f(x)^{p_{1}} d x\right\} .
$$


Next, by the reiteration result of Holmstedt [1], it suffices to consider the case $0<p_{0}<1,2 \leq p_{1}<+\infty$. It is at this point then the atomic decomposition of Chang and Fefferman [2] (also Cohen [4]) plays a crucial rôle. For each $k \in \mathbf{Z}_{+}$let

$\Omega_{k}=\left\{S f>2^{k}\right\}$,

$R_{0}=\left\{\right.$ all dyadic rectangles $R$ such that $\left.\left|R \cap \Omega_{0}\right| \leq|R| / 2\right\}$,

$R_{k}=\left\{\right.$ all dyadic rectangles $R$ such that $\left.\left|R \cap \Omega_{k-1}\right|>|R| / 2,\left|R \cap \Omega_{k}\right| \leq|R| / 2\right\}$, $k \geq 1$.

For a dyadic rectangle $R=I \times J$, let

$$
R^{+}=\left\{(y, t) \in \mathbf{R}_{+}^{2} \times \mathbf{R}_{+}^{2}: y \in R,|I|<t_{1}<2|I|,|J|<t_{2}<2|J|\right\} .
$$

Also $A_{k}^{+}=\bigcup_{R \in R_{k}} R^{+}$. Put

$$
f_{0}(x)=\sum_{k>0} \iint_{A_{k}^{+}} f(y, t) \Phi_{t}(x-y) d y \frac{d t_{1} d t_{2}}{t_{1} t_{2}} \equiv \sum_{k>0} b_{k}(x)
$$

and

$$
f_{1}(x)=\iint_{A_{0}^{+}} f(y, t) \Phi_{t}(x-y) d y \frac{d t_{1} d t_{2}}{t_{1} t_{2}}
$$

That $f=f_{0}+f_{1}$, in $S^{\prime}$, follows readily by taking Fourier transforms, since $\int_{0}^{\infty} \hat{\varphi}(u)^{2} d u / u=1$. To complete the proof, we consider two claims, namely,

$$
\begin{aligned}
\left\|f_{0}\right\|_{H^{p_{0}}}^{p_{0}} & \leq C \int_{\{S f>1 / 2\}} S f(x)^{p_{0}} d x \\
& \leq C\left(\int_{\{S f>1\}} S f(x)^{p_{0}} d x+\int_{\{S f \leq 1\}} S f(x)^{p_{1}} d x\right)
\end{aligned}
$$

and

$$
\left\|f_{1}\right\|_{H^{p_{1}}}^{p_{1}} \leq C \int_{\{S f \leq 1\}} S f(x)^{p_{1}} d x
$$

The proof of (1.2) is achieved by showing that for each $k$ the normalization of $b_{k}(x)$ given by $C b_{k}(x) / 2^{k}\left|\Omega_{k-1}\right|^{1 / p_{0}}$ yields a $p_{0}$-atom (in the sense of ChangFefferman [2]). This would imply that

$$
\left\|f_{0}\right\|_{H^{p_{0}}}^{p_{0}} \leq C \sum_{k>0} 2^{k p_{0}}\left|\Omega_{k-1}\right| \leq C \int_{\{S f>1 / 2\}} S f(x)^{p_{0}} d x
$$


and thus give (1.2). We need to verify that

(i) $\quad\left\|b_{k}\right\|_{L^{2}} \leq C 2^{k}\left|\Omega_{k-1}\right|^{1 / 2}$,

(ii) $\operatorname{supp} b_{k} \subseteq \tilde{\Omega}_{k-1}=\bigcup_{R \in R_{k}} 4 R$,

(iii) $\quad b_{k}=\sum_{R \in R_{k}} \iint_{R^{+}} f(y, t) \Phi_{t}(x-y) d y \frac{d t_{1} d t_{2}}{t_{1} t_{2}}=\sum_{R \in R_{k}} b_{k, R}$,

(iv) $\int b_{k, R}\left(x_{1}, x_{2}\right) x_{1}^{m} d x_{1}=\int b_{k, R}\left(x_{1}, x_{2}\right) x_{2}^{m} d x_{2}=0$ for all $m$ up to $\sim\left(1 / p_{0}-1\right)$,

(v) $\operatorname{supp} b_{k, R} \subseteq 4 R$,

(vi) $\quad b_{k, R}$ is in $C^{1}$ and $\left\|b_{k, R}\right\|_{L^{\infty}} \leq d_{R}\left(C 2^{k}\left|\Omega_{k-1}\right|^{1 / p_{0}}\right)$,

$$
\begin{aligned}
& \left\|\frac{\partial}{\partial x_{1}} b_{k, R}\right\|_{L^{\infty}} \leq d_{R} \frac{C 2^{k}\left|\Omega_{k-1}\right|^{1 / p_{0}}}{|I|^{m}}, \\
& \left\|\frac{\partial}{\partial x_{2}} b_{k, R}\right\|_{L^{\infty}} \leq d_{R} \frac{C 2^{k}\left|\Omega_{k-1}\right|^{1 / p_{0}}}{|J|^{m}}, \quad R=I \times J
\end{aligned}
$$

and, finally,

$$
\sum_{R \in \mathcal{R}_{k}} d_{R}^{2}|R| \leq C\left|\Omega_{k-1}\right|^{1-2 / p_{0}}
$$

The proof of these assertions is similar to that of [2, Theorem 1$]$, where it is done for $p_{0}=1$. Let us see that (i) holds. For this purpose pick $g \in L^{2}$ with $\|g\|_{L^{2}}=1$. Then

$$
\begin{aligned}
\left|\int b_{k}(x) g(x) d x\right|=\left|\iint_{A_{k}^{+}} f(y, t) g(y, t) d y \frac{d t_{1} d t_{2}}{t_{1} t_{2}}\right| & \\
\leq C \iint_{A_{k}^{+}}\left[\frac{1}{t_{1} t_{2}} \mid\left\{z \in \mathbf{R}^{2}: M\left(\chi_{\Omega_{k-1}}, z\right)>\frac{1}{2},\right.\right. & \left.\left.z \in \Omega_{k}^{c},(y, t) \in \Omega(z)\right\} \mid\right] \\
& \times|f(y, t)||g(y, t)| d y \frac{d t_{1} d t_{2}}{t_{1} t_{2}}
\end{aligned}
$$

where $M$ denotes the strong maximal function. By Tonelli's theorem and Schwartz's inequality this last integral does not exceed

$$
\begin{aligned}
\int_{\left\{M\left(\chi \Omega_{k-1}\right)>1 / 2\right\} \cap \Omega_{k}^{c}} S f(z) S g(z) d z & \leq\left(\int_{\left\{M\left(\chi \Omega_{k-1}\right)>1 / 2\right\} \cap \Omega_{k}^{c}} S f(z)^{2} d z\right)^{1 / 2}\|S g\|_{L^{2}} \\
& \leq C 2^{k}\left|\left\{M\left(\chi \Omega_{k-1}\right)>\frac{1}{2}\right\}\right|^{1 / 2} .
\end{aligned}
$$

Hence, by the strong maximal theorem,

$$
\left|\int b_{k}(x) g(x) d x\right| \leq C 2^{k}\left|\Omega_{k-1}\right|^{1 / 2}
$$


which gives (i). The proofs of (ii)-(v) are trivial. As for as for (vi) and (vii), clearly

$$
\begin{aligned}
\left\|b_{k, R}\right\|_{L^{\infty}} & \leq C \iint_{R^{+}}|f(y, t)| d y \frac{d t_{1} d t_{2}}{t_{1} t_{2}} /|R| \\
& \leq C|R|^{-1 / 2}\left(\iint_{R^{+}}|f(y, t)|^{2} d y \frac{d t_{1} d t_{2}}{t_{1} t_{2}}\right)^{1 / 2}
\end{aligned}
$$

and arguing as above we see that

$$
\begin{aligned}
\sum_{R \in R_{k}} \iint_{R^{+}}|f(y, t)|^{2} d y \frac{d t_{1} d t_{2}}{t_{1} t_{2}} & \leq C \int_{\left\{M\left(\chi \Omega_{k-1}\right)>1 / 2\right\} \cap \Omega_{k}^{c}} S f(z)^{2} d z \\
& \leq C 2^{2 k}\left|\Omega_{k-1}\right|
\end{aligned}
$$

After the proper normalization this gives (vi)-(vii), except for the estimates of the partial derivatives of $b_{k, R}$, which we leave for the reader to verify.

The proof of (1.3) is similar. For any function $h$ with $\|h\|_{L^{p_{1}}}=1$ we find, as above,

$$
\begin{aligned}
\left|\int f_{1}(x) h(x) d x\right| & \leq \iint_{A_{0}^{+}}|f(y, t)| d y \frac{d t_{1} d t_{2}}{t_{1} t_{2}} \\
& \leq C \int_{\Omega_{0}^{c}} S f(z) S h(z) d z \leq C\left(\int_{\Omega_{0}^{c}} S f(z)^{p_{1}} d z\right)^{1 / p_{1}}
\end{aligned}
$$

This implies (1.3) and completes the proof.

A few remarks about Theorem A: Let $\mathcal{M} f(x)=\sup _{(y, t) \in \Gamma(x)}\left|P_{t} * f(y)\right|$ be the nontangential maximal function. If we use the proof of the atomic decomposition, due to J. M. Wilson [10], which is based on the nontangential maximal function rather than on the square function, an argument entirely analogous to the one above gives

THEOREM $\mathrm{A}^{\prime}$. Let $0<p_{0}<p_{1}<+\infty$. Then

$$
K\left(t, f ; H^{p_{0}}, H^{p_{1}}\right) \approx K\left(t, \mathcal{M} f ; L^{p_{0}}, L^{p_{1}}\right) .
$$

Moreover, as an immediate consequence of Theorem $\mathrm{A}$ (or $\mathrm{A}^{\prime}$ ) we have the following result of $\operatorname{Lin}[8]$.

CoROllaRY B. Let $0<p_{0}<p_{1}<+\infty$ and $1 / p=(1-\theta) / p_{0}+\theta / p_{1}, 0<\theta<1$. Then $\left(H^{p_{0}}, H^{p_{1}}\right)_{\theta p}=H^{p}$ (with equivalent quasinorms).

Let us mention two simple applications of Corollary B.

In [6] it is shown that for any $p_{0}, 0<p_{0}<1$, and $f \in H^{p_{0}}\left(\mathbf{R}_{+}^{2} \times \mathbf{R}_{+}^{2}\right)$,

$$
\left|\hat{f}\left(\xi_{1}, \xi_{2}\right)\right| \leq C\left|\xi_{1}\right|^{1 / p_{0}-1}\left|\xi_{2}\right|^{1 / p_{0}-1}\|f\|_{H^{p_{0}}} .
$$

Let $d \mu\left(\xi_{1}, \xi_{2}\right)=d \xi_{1} d \xi_{2} /\left|\xi_{1}\right|\left|\xi_{2}\right|$, and let $T f\left(\xi_{1}, \xi_{2}\right)=\left|\xi_{1}\right|\left|\xi_{2}\right| \hat{f}\left(\xi_{1}, \xi_{2}\right)$. Then the above estimate means that $T$ is bounded from $H^{p_{0}}$ to $L^{p_{0, \infty}}(d \mu), 0<p_{0}<1$. On the other hand, by Plancherel's theorem, $T$ is bounded from $H^{2}$ into $L^{2}$. Hence, 
using Corollary B and the Marcinkiewicz interpolation theorem, we obtain

Proposition C (HARdy-LitTlewood ImBedding Theorem). Let $0<$ $p \leq 2$ and $f \in H^{p}\left(\mathbf{R}_{1}^{2} \times \mathbf{R}_{1}^{2}\right)$. Then

$$
\left(\iint\left|\hat{f}\left(\xi_{1}, \xi_{2}\right)\right|^{p}\left|\xi_{1}\right|^{p-2}\left|\xi_{2}\right|^{p-2} d \xi_{1} d \xi_{2}\right)^{1 / p} \leq C\|f\|_{H^{p}}
$$

In a similar way we can prove results concerning the modified Riesz potential $I_{\alpha}$ of order $\alpha$-that is, the operator given by the inverse Fourier transform of

$$
\left(I_{\alpha} f\right)^{\Upsilon}\left(\xi_{1}, \xi_{2}\right)=\hat{f}\left(\xi_{1}, \xi_{2}\right)\left|\xi_{1}\right|^{-\alpha}\left|\xi_{2}\right|^{-\alpha}, \quad 0<\alpha<+\infty .
$$

Proposition D. Let $0<p<q<+\infty, 0<\alpha<1 / p$, and $1 / q=1 / p-\alpha$. Then $\left\|I_{\alpha} f\right\|_{H^{q}} \leq C\|f\|_{H^{p}}$.

\section{REFERENCES}

1. J. Bergh and J. Löfström, Interpolation spaces: an introduction, Springer-Verlag, Berlin and New York, 1976.

2. S. Y. A. Chang and R. Fefferman, A continuous version of duality of $H^{1}$ and BMO on the bi-disc, Ann. of Math. (2)112 (1780), 179-201.

3. _ The Calderón-Zygmund decomposition on product domains, Amer. J. Math. 104 (1982), 445-468.

4. G. Cohen, $H^{(p, q)}$ : some new spaces of distributions with atomic decomposition, preprint.

5. R. Gundy and E. M. Stein, $H^{p}$ theory for the poly-disc, Proc. Nat. Acad. Sci. U.S.A. 76 (1979), 1026-1029.

6. B. Jawerth, R. Rochberg and G. Weiss, Commutator and other second order estimates in real interpolation theory, preprint.

7. B. Jawerth and A. Torchinsky, On a Hardy-Littlewood imbedding theorem, Michigan Math. J. 31 (1984), 131-137.

8. K. C. Lin, $H^{p}$ interpolation on the bi-disc, preprint.

9. K. G. Merryfield, $H^{p}$ spaces in poly-half spaces, Ph.D. Thesis, Univ. of Chicago, 1980.

10. J. M. Wilson, On the atomic decomposition for Hardy spaces, Pacific J. Math. 116 (1985), 201-207. 63130

Department of MAthematics, Washington University, St. Louis, Missouri

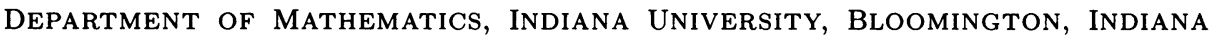
47405 